\title{
BRAQUIÓPODOS ARTICULADOS DE LA FORMACIÓN EL PASO, PALEOZOICO SUPERIOR, PRECORDILLERA ARGENTINA
}

\author{
Tristán SIMANAUSKAS' y Gabriela A. CISTERNA² \\ ' Departamento Científico de Geología, Museo de La Plata. Paseo del Bosque s/n. \\ 1900 La Plata, Argentina. \\ 2 Facultad de Ciencias Naturales e Instituto Miguel Lillo. Miguel Lillo 205. 4000 \\ San Miguel de Tucumán, Argentina
}

\begin{abstract}
Simanauskas, T. y Cisterna, G. 2001. Braquiópodos articulados de la Formación El Paso, Paleozoico Superior, Precordillera Argentina. [Articulate brachiopods of the El Paso Formation, Upper Paleozoic. Argentine Precordillera.] Revista Española de Paleontología, 16(2), 209-222. ISSN 0213-6937.
\end{abstract}

\begin{abstract}
This paper provides a review of the articulate brachiopod faunas from the El Paso Formation, CalingastaUspallata Basin, Upper Paleozoic of the San Juan province, Argentina. The brachiopod assemblage studied contains Rhipidomella? sp., Micraphelia indianae sp. nov., Tuberculatella peregrina (Reed, 1927), Aseptella aff. patriciae Simanauskas and Meristelloidea indet. The fauna of the El Paso Formation was described previously and included in the Rugosochonetes-Bulahdelia Biozone, assigned to the Early Carboniferous. However, the new records and its biostratigraphical relationships allow to conclude that the brachiopod faunas from the El Paso Formation, would be inside of the range Latest Carboniferous - Early Permian.
\end{abstract}

Keywords: Brachiopods, Upper Paleozoic, systematic paleontology, biostratigraphy, Argentina.

\section{RESUMEN}

En este trabajo se estudia la fauna de braquiópodos articulados de la Formación El Paso, cuenca CalingastaUspallata, Paleozoico tardío de la provincia de San Juan, Argentina. El conjunto faunístico analizado está integrado por Rhipidomella? sp., Micraphelia indianae sp. nov., Tuberculatella peregrina (Reed, 1927), Aseptella aff. patriciae Simanauskas y Meristelloidea indet. La fauna de la Formación El Paso fue previamente incluida en la Biozona de Rugosochonetes-Bulahdelia y asignada al Carbonífero temprano. Sin embargo, las nuevas determinaciones y reasignaciones, como así también las relaciones bioestratigráficas de los braquiópodos estudiados, sugieren que la Formación El Paso alcanzaría una edad más joven dentro del intervalo Carbonífero terminal- Pérmico temprano.

Palabras clave: Braquiópodos, Paleozoico Superior, paleontología sistemática, bioestratigrafía, Argentina.

\section{INTRODUCCIÓN}

La Formación El Paso aflora en el extremo sur de la Sierra de Barreal, aproximadamente $4 \mathrm{~km}$ al sudeste de la localidad homónima, en la provincia de San Juan (Fig. 1). Fue definida por Mésigos (1953), quién la ubicó, junto a las formaciones Hoyada Verde y El Retamo, en el Grupo San Eduardo, asignándolo al Misisipiense. Sin embargo, dicho autor sugiere que la Formación El Paso podría corresponder a la unidad más antigua de las reunidas en este grupo. Posteriormente Amos y Rolleri (1965), al revisar la estratigrafía de la cuenca, asignan el Grupo San Eduardo al Carbonífero medio. Taboada (1989), basándose fundamentalmente en el estudio de braquiópodos, define en esta unidad la Biozona de Rugosochonetes-Bulaludelia, a la que asigna una edad carbonífera temprana. Más recientemente González (1993), en una nueva interpretación estratigráfica de los depósitos marinos de la cuenca Calingasta-Uspallata, considera las formaciones Hoyada Verde y El Paso como miembros superior e inferior, respectivamente, de la Formación San Eduardo (Mésigos, 1953; nom. transl. González, 1993). Finalmente, Taboada (1997), siguiendo esta última propuesta, sostiene la presencia de la Biozona de Rugosochonetes-Bulahdelia en el Miembro El Paso.

Cisterna (1997) analiza en forma preliminar la 


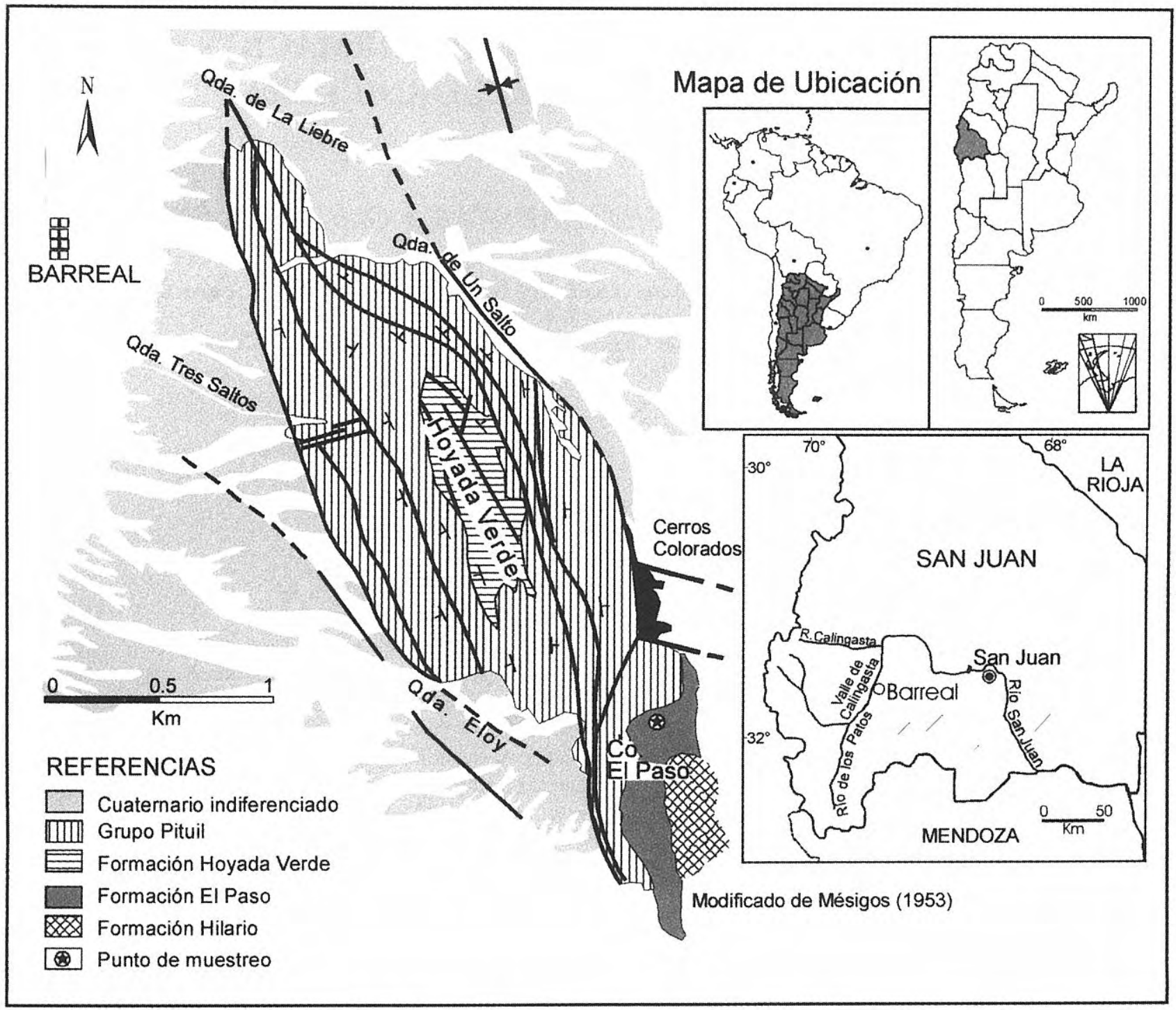

Figura 1. Mapa de ubicación y distribución de los afloramientos de la Formación El Paso. Location map and occurrence of the El Paso Formation outcrops.

distribución y composición de la fauna de la Formación El Paso, considerando que la misma representaría una unidad cronológicamente equivalente o ligeramente más joven que la Formación Hoyada Verde.

De los recientes estudios litofaciales, paleoambientales y sistemáticos realizados por los autores en la Formación El Paso (Martínez et al., 1998), se dan a conocer, en la presente contribución, las nuevas determinaciones y reasignaciones de los braquiópodos articulados y las implicaciones bioestratigráficas que surgen de las mismas.

La totalidad del material aquí estudiado procede de la misma localidad (Quebrada de Eloy, extremo sur de la sierra de Barreal, aproximadamente $3,5 \mathrm{~km}$ al sudeste del pueblo de Barreal, provincia de San Juan, Argentina) y se encuentra depositado en las colecciones del Instituto de Paleontología, sección invertebrados de la Fundación Miguel Lillo, San Miguel de Tucumán (IPI) y del Departamento Científico de Geología del Museo de La Plata (DCG-MLP).

\section{CONTENIDO FAUUNÍSTICO DE LA FORMACIÓN EL PASO}

La fauna de la Formación El Paso presenta una rica asociación de invertebrados marinos fósiles, vinculada a niveles de diamictitas que han sido interpretadas como de origen glacigénico (Mésigos, 1953; Amos y Rolleri, 1965; González, 1990; Martínez et al., 1998, entre otros).

En la parte superior del perfil de esta unidad estratigráfica, se han reconocido dos intervalos faunísticos ubicados inmediatamente por debajo y por encima del principal nivel de diamictitas (Fig. 2).

El intervalo faunístico inferior está representado fundamentalmente por braquiópodos, gasterópodos, escasos bivalvos y fragmentos de crinoideos. Asimismo, en niveles concrecionales se asocian también a esta fauna nautiloideos ortocónicos y conularios. Este intervalo corresponde a un paquete de aproximadamente $30 \mathrm{~m}$, en el cual la fauna adquiere una distribución continua a lo largo de todo su espesor. Sin embargo, se han delimitado 
8 puntos de muestreo, teniendo en cuenta los principales cambios faunísticos dentro del intervalo. Del punto 1 al 4, la asociación faunística está dominada fundamentalmente por braquiópodos (Rhipidomella? sp., Micraphelia indianae sp. nov. y Tuberculatella peregrina (Reed, 1927)), acompañados por gasterópodos y bivalvos. Entre los puntos 2 y 3 se localiza un nivel concrecional que exhibe una fauna más diversificada de gasterópodos, bivalvos, nautiloideos ortocónicos y conularios (Fig. 2).

A partir del punto 4 se observa un cambio en la composición faunística. La diversidad comienza a aumentar en forma paulatina siendo máxima en el punto 8 en el cual los braquiópodos están presentes con el mayor número de especies reconocidas en el intervalo, sumándose, a las ya citadas, Aseptella aff. patriciae Simanauskas y Meristelloidea indet.

El intervalo fosilífero superior corresponde al punto 9 del perfil y está representado por un conjunto de invertebrados marinos y tallos vegetales. Entre los primeros, son dominantes los braquiópodos, con Rhipidomella? sp. más abundante que en los niveles anteriores, Micraphelia indianae sp. nov. y escasos braquiópodos inarticulados, estando la fauna asociada representada por gasterópodos y corales.

\section{EDAD DE LOS BRAQUIÓPODOS Y CONSIDERACIONES BIOESTRATIGRÁFICAS}

Sobre la base de la identificación de Rugosochonetes gloucesterensis (Cvancara) y de Bulahdelia cf. myallensis Roberts, Taboada (1989) define en la Formación El Paso la Biozona de Rugosochonetes-Bulahdelia y le asigna una edad carbonífera inferior tardía (Visense tardíoNamuriense temprano). Desde el punto de vista bioestratigráfico, la citada biozona es ubicada por su autor inmediatamente por debajo de la Biozona de Levipustula en Argentina (Taboada 1989, 1997). Según el mismo, la edad del límite superior de la Biozona de Rugosochonetes-Bulahdelia queda definida por la edad de la base de la Biozona de Levipustula en Australia (Namuriense temprano), mientras que la edad del límite inferior estaría dada por la edad de la Biozona de Rhipidomella fortimuscula en Australia (Viseense tardío). Resulta importante destacar que no existe relación física entre la Formación El Paso y la Formación Hoyada Verde en la sierra de Barreal, lo cual impide establecer las relaciones de infra o suprayacencia entre la fauna aquí estudiada y la fauna de la Biozona de Levipustula que caracteriza a la Formación Hoyada Verde.

La Biozona de Rugosochonetes-Bulahdelia ha sido también mencionada en la cuenca Tepuel-Genoa de Patagonia, en donde Taboada (1997, págs. 223-224) señala: "la Cronozona de Rugosochonetes-Bulahdelia es indicada para sedimentitas glacigénicas estériles ubicadas en los 200 metros inferiores de la Formación Pampa de Tepuel, en la Patagonia extraandina". Sin embargo, en el mismo párrafo, dicho autor continúa refiriéndose a la misma unidad en términos dé una unidad bioestratigráfica

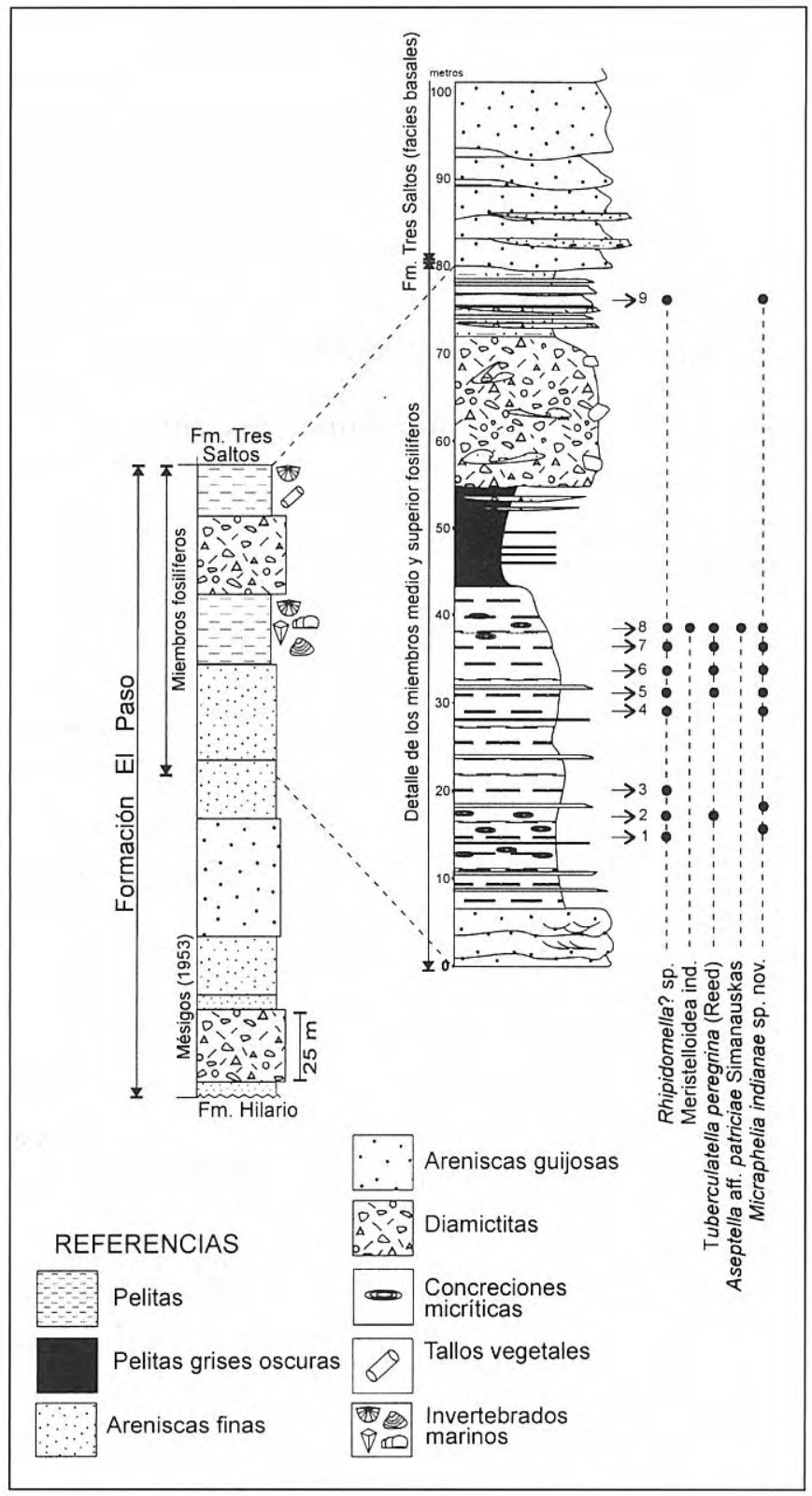

Figura 2. Perfil esquemático de la Formación El Paso y detalle del intervalo fosilífero, indicando la distribución vertical de los braquiópodos estudiados.

Schematic section of El Paso Formation and detail of the fossiliferous interval, indicating the vertical distribution of the studied brachiopods.

(biozona). Asimismo, cabe agregar que desde su definición original (Taboada, 1989), la Biozona de Rugosochonetes-Bulahdelia ha sido citada por su autor, de manera indistinta, como Zona o como Biozona (como ejemplo ver Taboada, 1989, pág. 115).

Las nuevas determinaciones y reasignaciones basadas en el abundante material coleccionado por los autores en diferentes niveles de la Formación El Paso, así como el hallazgo de esta fauna en otras localidades, exigen un replanteo profundo de su edad y sus relaciones bioestratigráficas. 
Se han identificado dos asociaciones de braquiópodos presentes en esta formación y en otras localidades, a saber: Aseptella-Tuberculatella y Rhipidomella?Micraphelia.

Tuberculatella es un género distribuido entre el Carbonífero tardío y el Pérmico temprano (Brunton et al., 1995). Tuberculatella peregrina se asemeja tanto a Tuberculatella laevicaudata (Amos) del Pérmico de Patagonia, cuenca Tepuel-Genoa (Simanauskas, 1996c), como a Tuberculatella tubertella Waterhouse, 1982, del Carbonífero tardío de Tailandia, y puede ser interpretada como una forma morfológicamente intermedia entre ambas, lo cual no debe interpretarse como evidencia de que la especie sanjuanina tenga una posición temporal intermedia entre ambas. Del género Aseptella se han descripto hasta el presente sólo tres especies, dos del Carbonífero de España (Aseptella asturica Martínez Chacón y Winkler Prins, 1977 y Aseptella beetsi Winkler Prins y Martínez Chacón, 1998) y Aseptella patriciae Simanauskas, 1996b, del Pérmico temprano de Patagonia. Aseptella aff. patriciae, integrante de la fauna de El Paso, muestra similitudes, obviamente, con la especie patagónica.

Tanto Tuberculatella laevicaudata como Aseptella patriciae, integran en la cuenca Tepuel-Genoa la Biozona de Tuberculatella de edad sakmariense temprana. Esta biozona se ubica, en la citada cuenca, por encima de la Biozona de Lanipustula y por debajo de la Faúnula de Costatumulus (Simanauskas y Sabattini, 1997).

La asociación Aseptella-Tuberculatella también ha sido registrada en otras localidades de la cuenca Calingasta-Uspallata. Recientemente, Lech et al. (1998) dan a conocer en el nivel fosilífero 1 de la Formación Casa de Piedra (Banchig et al., 1997), en la Sierra del Tontal, la presencia de braquiópodos asignados en este trabajo a Aseptella aff. patriciae y Tuberculatella peregrina. En forma similar a lo que ocurre en la cuenca Tepuel-Genoa, inmediatamente por encima de los mismos, Lech et al. (1998) describen e ilustran Cancrinella? y Septosyringothyris aff. S. saltensis Lech, elementos comunes en el Pérmico temprano de Argentina.
El género Micraphelia Cooper y Grant, está representado hasta el presente sólo en Pérmico tardío (Guadalupiense) de Tejas y con dudas en el Pérmico tardío de Malasia. Micraphelia indianae sp. nov., por sus caracteres externos, se asemeja a la especie tipo del género, Micraphelia scitula Cooper y Grant, 1969, como así también a Micraphelia pumilis Cooper y Grant, 1975, también del oeste de Tejas (Formación Bone Spring). Sin embargo, de las especies del oeste de Tejas, Micraphelia subalata Cooper y Grant, 1975, proveniente del Miembro Hegler de la Formación Bell Canyon, es la que presenta mayores afinidades en sus caracteres internos con Micraphelia indianae sp. nov.

En la Formación El Paso, Rhipidomella? sp. es el braquiópodo más abundante y presenta gran afinidad con especies pérmicas dada su morfología y tamaño (Rhipidomella cordialis Grant del Pérmico de Tailandia, Rhipidomella cora (d'Orbigny) del Pérmico de Perú y Rhipidomella sp. del Pérmico de Indonesia).

Rhipidomella? sp. y Micraphelia indianae sp. nov. han sido recientemente halladas por los autores en la Formación Agua del Jagüel (Precordillera de Mendoza), en los niveles pelíticos de la sección inferior ubicados inmediatamente por encima de niveles de diamictitas. Estas últimas serían litofacialmente comparables con aquéllas de la Formación El Paso (Martínez, com. pers.). Asimismo, cabe agregar que los niveles de la sección superior de la Formación Agua del Jagüel son portadores de la fauna que caracteriza la Biozona de Costatumulus amosi de edad pérmica temprana (Taboada, 1998).

En conclusión, los braquiópodos presentes en la Formación El Paso resultan importantes elementos de correlación dentro de la cuenca Calingasta-Uspallata, como así también con la cuenca Tepuel-Genoa. Por su ubicación estratigráfica y afinidades, esta fauna sería más joven que aquélla que integra la Biozona de Levipustula, tal como es considerada actualmente (NamurienseWestfaliense? en Argentina, Taboada, 1997; González, 1999, o bien restringida al Namuriense en Australia, Roberts et al., 1995). Asimismo, se ha señalado que en otras localidades de la cuenca Calingasta-Uspallata (Agua del Jagüel y Casa de Piedra), las asociaciones faunísticas

Figura 3. a-h. Rhipidomella? sp. a, molde externo de valva ventral, external mould of ventral valve, IPI $2808, x 5,5$. b, molde interno de un ejemplar articulado, vista dorsal, internal mould of an articulate specimen, dorsal view, IPI 2820 , x5. c, molde externo de valva dorsal, external mould of dorsal valve, IPI 2807, x5,5. d, molde interno de valva ventral, internal mould of ventral valve, IPI 2833, x5. e, molde interno de valva ventral, internal mould of ventral valve, IPI 2832, x5. f-g, molde interno de valva dorsal con su contramolde externo, internal mould of dorsal valve and its external mould, IPI 2816a-b, x5,5. h, molde interno de valva dorsal, internal mould of dorsal valve, IPI $2818 \mathrm{a}$, x5. i-s. Micraphelia indianae sp. nov. i, k, réplica en látex del molde interno y externo de la valva dorsal, latex cast of the internal and external mould of the dorsal valve, DCG-MLP 303b Holotipo, x3,5. j, réplica en látex del molde interno de la valva dorsal, latex cast of the internal mould of the dorsal valve, DCG-MLP 306f, x3,5. 1, molde interno de valva dorsal, internal mould of dorsal valve, DCG-MLP 307a, x3. m, réplica en látex del molde externo de la valva dorsal, latex cast of the external mould of the dorsal valve, DCG-MLP 306c, x3,5. n, réplica en látex del molde interno de la valva ventral, latex cast of internal mould of the ventral valve, DCG-MLP 305d, x2,5. o, réplica en látex del molde externo de la valva dorsal, latex cast of external mould of the dorsal valve, IPI 303d, x3,5. p, réplica en látex del molde externo de la valva ventral, latex cast of external mould of the ventral valve, DCG-MLP 303c, x3,5. q, r, réplica en látex del molde externo de las valvas ventral y dorsal, respectivamente, latex cast of external mould of the dorsal and ventral valves respectively, DCG-MLP 306h, x6,5. s, réplica en látex del molde interno de la valva ventral, latex cast of the internal mould of ventral valve, DCG-MLP 305c, x3. 

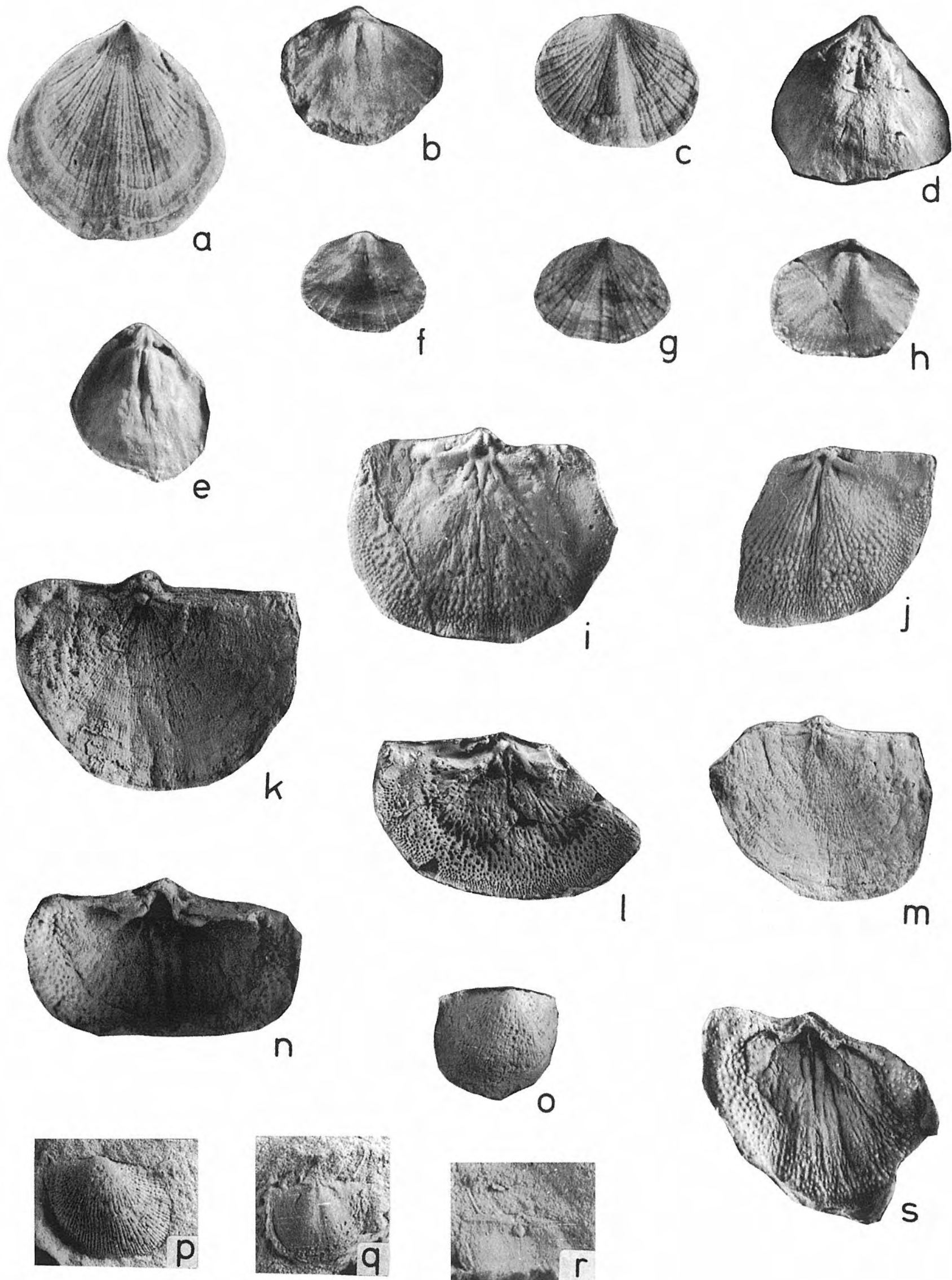
registradas en El Paso estarían por debajo de la Biozona de Costatumulus amosi. De este modo, considerando la afinidad de los braquiópodos analizados y sus relaciones bioestratigráficas, los mismos tendrían una edad comprendida en el intervalo Carbonífero terminalPérmico temprano.

\section{DESCRIPCIONES SISTEMÁTICAS}

ORDEN ORTHIDA Schuchert y Cooper, 1932

SUBORDEN DALMANELLIDINA Moore, 1952

Superfamilia DALMANELLOIDEA Schuchert, 1913

Familia Rhipidomellidae Schuchert, 1913

Sufamilia Rhipidomellinae Schuchert, 1913

Género Rhipidomella Oehlert, 1890

Especie tipo: Terebratula michelini Léveillé, 1835, descrita para el Carbonífero temprano de Europa.

Rhipidomella? sp.

Fig. 3a-h, Tab. 1

Localidad: Quebrada de Eloy, extremo sur de la sierra de Barreal, aproximadamente $3,5 \mathrm{~km}$ al sudeste del pueblo de Barreal, provincia de San Juan, Argentina.

Posición Estratigráfica: Parte superior del perfil de la Formación El Paso, intervalos faunísticos inferior y superior, niveles 1 al 9.

Material: Diez moldes externos de valvas ventrales, IPI 2801 (N3), 2808 (N5), 2812 (N1-2), 2813, 2814 (N4-5), 2819, 2821, 2822, 2823 (N9), 2829 (N8); seis moldes externos de valvas dorsales, IPI 2803 (N2), 2805, 2806, 2807 (N4), 2824, 2828 (N9); nueve moldes internos de valvas ventrales algunos de ellos con su contramolde, IPI 2800 (N3), 2802 (N2), 2809a-b (N6), 2811 (N1-2), 2815 (N5), 2820, 2827 (N9), 2830 (N8), 2832 (N1); dos moldes internos de valvas dorsales con sus contramoldes, IPI 2816ab (N6), 2818a-b (N9); un molde interno correspondiente a un ejemplar articulado, IPI 2831(N1). Completan el material algunos ejemplares deformados, IPI 2804 (N2), 2810a-b (N7), 2825, 2826 (N9).

\section{Descripción}

Concha planoconvexa, notablemente pequeña para el género (ver Tab. 1); contorno subtriangular; anchura aproximadamente igual a la longitud o levemente menor; línea cardinal considerablemente estrecha en comparación con la característica del género. No se han observado las características de la interárea. Valva ventral escasamente convexa, alcanzando la máxima convexidad a la mitad de la longitud valvar. Umbón agudo y débilmente incurvado. Valva dorsal plana con una región umbonal ligeramente prominente y un seno débil pero bien definido que se inicia en la región umbonal. Ambas valvas presentan una ornamentación compuesta de finas costillas radiales, que se subdividen en la región umbonal y cerca del margen anterior, en donde alcanzan una densidad de aproxima-

\begin{tabular}{|c|c|c|}
\hline $\mathrm{IPI}$ & $\mathrm{A}$ & $\mathrm{L}$ \\
\hline 2800 & 5 & 5,5 \\
\hline 2801 & 4 & 4 \\
\hline 2802 & 4 & 4 \\
\hline 2805 & 5 & 5 \\
\hline 2806 & 5 & 5 \\
\hline 2807 & 5 & 4,5 \\
\hline 2808 & 7 & 7 \\
\hline 2809 & 5 & 4,5 \\
\hline 2812 & 3,5 & 4 \\
\hline 2813 & 5 & 6 \\
\hline 2814 & 6 & 7 \\
\hline 2816 & 4 & 4 \\
\hline 2818 & 5 & 4 \\
\hline 2819 & 8 & 5 \\
\hline 2820 & 6 & 5 \\
\hline 2821 & 6 & 5 \\
\hline 2822 & 4 & 4 \\
\hline 2824 & 4 & 4 \\
\hline 2828 & 6 & 6 \\
\hline 2829 & 6 & 6 \\
\hline 2830 & 5 & 5 \\
\hline
\end{tabular}

Tabla 1. Dimensiones en $\mathrm{mm}$ de Rhipidomella? sp. A: anchura; L: longitud.

Measurements in $\mathrm{mm}$ of Rhipidomella? sp. A: width; L: length.

damente 5 costillas por mm (medida tomada sólo en unos pocos ejemplares). La superficie exhibe también conspicuas líneas de crecimiento concéntricas, cuya densidad alcanza un valor de aproximadamente 15 líneas $/ \mathrm{mm}$ en la mitad de la longitud de la concha. Hacia la región media-anterior de la concha, algunas de ellas se tornan más prominentes que las restantes. El interior de la valva ventral presenta un campo muscular conspicuo, dividido en dos áreas de contorno ovalado por medio de una elevación (ridge) central que se proyecta anteriormente más allá del campo muscular. Otros caracteres ventrales internos no están preservados. El interior de la valva dorsal exhibe un par de braquióforos cortos y fuertemente divergentes. El campo muscular es pequeño y de contorno subcuadrado. Las características del proceso cardinal como así también otros caracteres internos no se han observado.

\section{Discusión}

El conjunto de caracteres diagnósticos observados en el material estudiado concuerdan en términos muy generales con aquellos señalados para las especies del género Rhipidomella. Sin embargo, se ha mantenido una designación genérica dudosa debido a la ausencia del perfil dorsibiconvexo que caracteriza a Rhipidomella, así como también debido a la pobre preservación de los caracteres internos en el material analizado.

Rhipidomella? sp. aquí descripta es una forma extremadamente pequeña, de contorno subtriangular, con una línea cardinal estrecha y conspicuas líneas de crecimiento concéntricas, carácter no observado en 


\begin{tabular}{|l|c|c|}
\hline DCG-MLP & $\mathrm{A}$ & $\mathrm{L}$ \\
\hline $302 \mathrm{~b}$ & 16 & 14 \\
\hline $303 \mathrm{a}$ & 4 & 3 \\
\hline $303 \mathrm{~b}$ & 14,7 & 10,4 \\
\hline $303 \mathrm{c}$ & 6,3 & 5,2 \\
\hline $303 \mathrm{~d}$ & 8 & 6 \\
\hline $304 \mathrm{a}$ & 3 & 2 \\
\hline $304 \mathrm{c}$ & 14,6 & 11 \\
\hline $304 \mathrm{~d}$ & 6,4 & 4 \\
\hline $304 \mathrm{e}$ & 8,4 & 6 \\
\hline $304 \mathrm{f}$ & 16 & 10 \\
\hline $305 \mathrm{c}$ & 15 & 11,5 \\
\hline $306 \mathrm{a}$ & 7 & 5,6 \\
\hline $306 \mathrm{~b}$ & 15 & 7,4 \\
\hline $306 \mathrm{e}$ & 4,7 & 4 \\
\hline $306 \mathrm{f}$ & 12 & 8,5 \\
\hline $306 \mathrm{~g}$ & 12 & 9 \\
\hline $306 \mathrm{~h}$ & 3 & 2,3 \\
\hline $307 \mathrm{~b}$ & 10,4 & 7,6 \\
\hline $307 \mathrm{c}$ & 16 & 10 \\
\hline $307 \mathrm{~d}$ & 12 & 9 \\
\hline $311 \mathrm{a}$ & 5,6 & 4,3 \\
\hline $311 \mathrm{~b}$ & 7 & 5 \\
\hline $311 \mathrm{c}$ & 8 & 6 \\
\hline $311 \mathrm{~d}$ & 8,2 & 6 \\
\hline $311 \mathrm{e}$ & 8 & 6,5 \\
\hline $311 \mathrm{f}$ & 8 & 5,6 \\
\hline $311 \mathrm{~g}$ & 9 & 6 \\
\hline $311 \mathrm{~h}$ & 10 & 7 \\
\hline $311 \mathrm{i}$ & 12 & 9 \\
\hline $311 \mathrm{j}$ & 13,6 & 9 \\
\hline $311 \mathrm{k}$ & 14 & 10,6 \\
\hline & & \\
\hline
\end{tabular}

Tabla 2. Dimensiones en $\mathrm{mm}$ de Micraphelia indianae sp. nov. A: anchura; L: longitud.

Measurements in mm of Micraphelia indianae $s p$. nov. A: width; L: length.

ninguna otra especie descrita hasta el momento en el género Rhipidomella. No obstante, futuras colecciones permitarían precisar su condición genérica como así también el establecimiento de una nueva especie.

El escaso tamaño de Rhipidomella? sp., que se aleja bastante del tamaño promedio que exhiben el resto de las especies que integran el género Rhipidomella, parecería ser un carácter inherente a especies descritas en niveles del Pérmico, en donde se han identificado las formas de menores dimensiones. Dentro de este grupo, Rhipidomella cordialis Grant del Pérmico de Tailandia, una de las formas más pequeñas reconocidas en el género, comparte este carácter con la especie argentina. Asimismo, algunos de los ejemplares figurados por Grant (1976, pág. 37, lám. 2, figs. 31-41; lám. 3, figs. 1-53), exhiben un contorno aproximado al de nuestra especie. Sin embargo, se trata de una forma fuertemente biconvexa a diferencia de Rhipidomella? sp.

Rhipidomella cora (d'Orbigny), descrita para el Pérmico temprano de Perú (Newell et al., 1953, pág. 91, lám. 16, figs.1a-8), si bien pequeña, duplica en tamaño a Rhipidomella? sp. Sin embargo, ambas especies son comparables en el contorno ligeramente sub-triangular de sus conchas, como así también en las características de la ornamentación.

Archbold (1981, pág. 8, lám. 1, figs. 3-4), describe para el Pérmico de Indonesia una valva ventral asignada a Rhipidomella sp. Si bien se trata de una forma relativamente pequeña, el contorno de la misma es notablemente más circular que el de Rhipidomella? sp.

Las especies del Carbonífero de Australia, Rhipidomella fortimuscula Cvancara (1958), Rhipidomella australis Maxwell (1961) y Rhipidomella michelini (Léveillé, 1835), se diferencian ampliamente de la especie argentina, tanto en su morfología externa como en su tamaño, ya que se trata de formas mucho más grandes. Fundamentalmente, Rhipidomella fortimuscula, que además exhibe un campo muscular ventral de gran dimensión en relación con el tamaño de la concha.

ORDEN PRODUCTIDA Sarytcheva y Sokolskaja, 1959

SUBORDEN CHONETIDINA Muir-Wood, 1955

Superfamilia CHONETOIDEA Bronn, 1862

Familia Rugosochonetidae Muir-Wood, 1962

Subfamilia Undulellinae Cooper y Grant, 1975

Género Micraphelia Cooper y Grant, 1969

Especie tipo: Micraphelia scitula Cooper y Grant, 1969, Bell Canyon Formation, Pérmico Inferior de Tejas.

\section{Diagnosis}

Rugosochonétidos pequeños, semielípticos a subrectangulares; la línea cardinal usualmente igual o levemente menor y ocasionalmente mayor que la anchura; moderada a fuertemente cóncavo-convexos; comisura anterior sin pliegue; superficie lisa o pseudocapilada cuando esta decorticada; espinas oblicuas a perpendiculares. Interior de la valva ventral con dientes moderadamente grandes y una gruesa callosidad apical; septo medio corto, aristas anteriores al septo de desarrollo variable, usualmente moderadamente gruesas alcanzando la mitad de la valva. Interior de la valva dorsal con un proceso cardinal grande y ancho; anderidios delicados y destacados; septo medio corto y delgado; septos accesorios usualmente cortos y anteriormente emplazados; aristas braquiales anterolateralmente engrosadas y espinosas.

Distribución geográfica y estratigráfica: Pérmico inferior, Sakmariense-Kunguriense de Tejas, América del Norte.

\section{Discusión}

En conchas pertenecientes a Micraphelia y Undulella, ambos géneros de la Subfamilia Undulellinae, se observa la presencia de septos accesorios (Cooper y Grant 1975, lámina 493 figuras 10, 18 a 20, 27 a 29 y 36; lámina 492 figuras 8 y 28). Se trata de hileras de endoespinas de desarrollo variable más elevadas que el resto. Estas estructuras flanquean un área central ubicada a ambos lados y por delante del septo medio. Este tipo de septos accesorios se encuentran mejor desarrollados en las 
conchas de los géneros de la Subfamilia Quinquenellinae Archbold (Racheboeuf, 1998).

\section{Micraphelia indianae sp.nov. Figs. 3i-s, 4, Tab. 2}

1989 Rugosochonetes gloucesterensis (Cvancara), Taboada, 124-125, lám. 2, figs. 10-11.

1989 Rugosochonetes sp. A., Taboada, 126, lám. 2, figs. 5-6. 1989 Rugosochonetes sp. B, Taboada, 126-127, lám. 2, figs. 7-8.

1990 Rugosochonetes gloucesterensis, Azcuy et al., fig. 2 (5).

Holotipo: un molde externo e interno de una valva dorsal, DCG-MLP 303 b, Fig. 3i, k.

Localidad tipo: Quebrada de Eloy, extremo sur de la sierra de Barreal, aproximadamente $3,5 \mathrm{~km}$ al sudeste del pueblo de Barreal, provincia de San Juan, Argentina.

Posición estratigráfica: Parte superior del perfil de la Formación El Paso, intervalo faunístico inferior y superior, niveles 1 a 9 .

Material: 9 moldes externos de valvas ventrales (DCGMLP 302 b; 303 a y b; 304 h, i; 306 j, k, y 1; 307 d); 22 moldes externos de valvas dorsales (DCG-MLP304 a, b, c, d, e, f, g; 305 e y f; 306 c, d, e, f, g, h, i; 307 b; 311 f, g, h, i, j); 4 moldes internos de valvas dorsales (DCG-MLP 302 a; 305 d, 311 a y d); 6 moldes internos de valvas ventrales (DCG-MLP 305 a, b, c; 307 c; 311 b y c); un molde interno y externo de valva ventral (DCG-MLP 303 c) y cuatro moldes externos e internos de valvas dorsales (DCG-MLP 303 b; 303 d; 306 a y b; 307 a); moldes internos y externos de un ejemplar completo (DCG-MLP 311e); una valva dorsal preservada (DCG-MLP 308 1) y una concha completa preservada (DCG-MLP 308 k).

Derivatio nominis: Dedicada a Indiana Simanauskas.

\section{Diagnosis}

Concha mediana a grande para el género, moderada a marcadamente cóncavo-convexa, semicircular a subcuadrangular; línea cardinal levemente menor que la anchura, la cual se mide en la mitad posterior de la concha. Espinas cardinales cirtomorfas intraversas, formando un ángulo inicial entre $50^{\circ}$ y $80^{\circ}$ con la línea cardinal (Fig. 4).

Shell medium to large for the genus, moderately to strongly concave-convex, semicircular to sub-square; hinge line slightly shorter than the maximum width, which is measured at posterior half of the shell. Cardinal spines cyrtomorph intraversed, forming an angle of about $50^{\circ}$ and $80^{\circ}$ with the hinge line (Fig. 4).

\section{Descripción}

Morfología externa: La concha es pequeña, el ejemplar más grande observado mide $16 \mathrm{~mm}$ de anchura y $14 \mathrm{~mm}$ de longitud (ver Tab. 2). El contorno es semicircular a

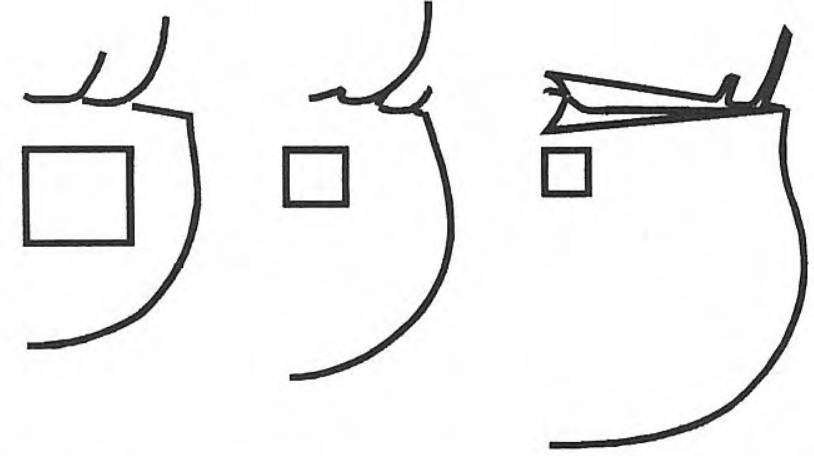

Figura 4. Dibujos realizados con cámara clara de la morfología de las espinas cardinales de Micraphelia indianae sp. nov. basados en los ejemplares DCG-MLP 306 h, 311 b y 311 i, de izquierda a derecha, respectivamente. El cuadrado representa $1 \mathrm{~mm}^{2}$.

Sketch with light camera about the morphology of the cardinal spines in Micraphelia indianae $s p$. nov., specimens DCG-MLP $306 \mathrm{~h}, 311$ b y $311 \mathrm{i}$, from left to right, respectively. The square represents $1 \mathrm{~mm}^{2}$.

subcuadrangular, la longitud equivale a tres cuartos de la anchura. La línea cardinal es ligeramente menor que la anchura, la cual se mide en la mitad de la longitud de la concha. Los extremos cardinales son obtusos y en algunos ejemplares redondeados. El perfil de la concha es moderada a marcadamente cóncavo-convexo. La interárea de la valva ventral es alta, casi el doble que la de la valva dorsal. Nototirio y quilidio desarrollados. Sobre el margen posterior de la valva ventral se han observado hasta 8 bases de espinas cardinales a cada lado del umbón (Fig. 4). El ángulo inicial varía entre $50^{\circ}$ y $80^{\circ}$ con respecto a la línea cardinal. En los ejemplares juveniles, las espinas son marcadamente cirtomorfas intraversas; parten del margen posterior en dirección posterolateral y luego se curvan posteriormente. En los ejemplares adultos, las espinas más grandes suelen ser algo más ortomorfas. Las valvas son lisas con líneas de crecimiento, más marcadas sobre las aurículas. Las valvas están comúnmente decorticadas y presentan el aspecto pseudocapilado característico.

Interior de la valva ventral: $\mathrm{El}$ interior de la valva ventral presenta un septo medio alto y corto, aproximadamente un séptimo de la longitud valvar. En el extremo anterior del septo, a ambos lados, surgen dos aristas elevadas, paralelas entre sí extendiéndose aproximadamente hasta la mitad de la longitud de la valva. A ambos lados del septo se ubican las impresiones de los músculos aductores, alargadas, que están rodeadas por las impresiones de los músculos diductores, grandes y redondeadas, las cuales se extienden hasta casi la mitad de la longitud de la valva. El resto de la valva esta tapizado de endoespinas, grandes en el borde lateral del disco, disminuyendo de tamaño hacia el margen comisural lateral y anterior.

Interior de la valva dorsal: El interior de la valva dorsal presenta un proceso cardinal grande, bilobado, con un 
alvéolo (pit) amplio en su base. A ambos lados del proceso se observan las aristas fosetales gruesas y curvadas, las cuales se extienden casi hasta la mitad del ancho de la línea cardinal. Por delante del alvéolo se desarrolla un septo medio corto, el cual se extiende hasta menos de la mitad de la longitud de la valva. Este septo es distalmente muy angosto y poco elevado. Los anderidios son largos, aproximadamente la mitad del septo medio. Las impresiones musculares están bien marcadas; las de los aductores anteriores son suavemente elevadas y redondeadas y las de los aductores posteriores son profundas y en forma de gota. En los ejemplares juveniles se observan dos septos accesorios a ambos lados del septo medio, los cuales se extienden por más de la mitad de la longitud de la valva; las aristas braquiales están formadas por endoespinas grandes formando un arco a ambos lados de los septos laterales; el área comprendida entre las aristas braquiales presenta hileras radiales de endoespinas. A diferencia de los juveniles, en los ejemplares adultos, los septos accesorios son menos conspicuos pero visibles; el extremo anterior de la arista braquial se curva hacia la región posterior sobre el área correspondiente a los septos accesorios y el área comprendida entre las aristas braquiales presenta escasas endoespinas. El resto de la concha, tanto en ejemplares adultos como juveniles, presenta hileras radiales de endoespinas que, al igual que en el interior de la valva ventral, disminuyen de tamaño hacia la comisura.

\section{Discusión}

Sobre la base de escaso material, Taboada (1989) asigna los conétidos de la Formación El Paso a Rugosochonetes gloucesterensis (Cvancara), Rugosochonetes sp. A. y Rugosochonetes sp. B. La precisa descripción de los conétidos realizada por este autor, permite reasignar el material estudiado a Micraphelia indianae sp. nov. El material asignado por Taboada a Rugosochonetes gloucesterensis (Cvancara) presenta el mismo contorno, perfil y dimensiones que Micraphelia indianae sp. nov., además del septo medio corto en la valva dorsal y las características del interior de la valva ventral. Las finas costillas características de Rugosochonetes, probablemente hayan sido confundidas con el aspecto pseudocapilado característico de Micraphelia. Esto puede inferirse de la descripción que hace Taboada (1989, página 126) de Rugosochonetes sp. A sic: "En las extremidades postero-laterales la ornamentación radial está aparentemente ausente."

\section{Comparaciones}

Micraphelia indianae sp. nov. se asemeja a la especie tipo del género, Micraphelia scitula, en el contorno y perfil de la concha y en la forma de las aristas fosetales. A Micraphelia pumilis Cooper y Grant, 1975 (pág. 1292, lám. 493, figs. 1-10), también del oeste de Tejas (Formación Bone Spring), se asemeja en el contorno y en el aspecto externo de la concha, pero difiere en el aspecto del interior de la valva dorsal. Los interiores de ambas valvas de Micraphelia indianae sp. nov. son muy semejantes a los de Micraphelia subalata Cooper y Grant, 1975 (pág. 1294), especie con la que presenta mayores afinidades. Esta semejanza es mayor aún con el material ilustrado de Micraphelia subalata, proveniente del Miembro Hegler de la Formación Bell Canyon, el cual presenta un contorno semicircular a subrectangular, con la línea cardinal de menor extensión que la anchura (Cooper y Grant, 1975, lám. 493, figs. 24 a 29).

\section{SUBORDEN PRODUCTIDINA Waagen, 1883}

Superfamilia PRODUCTOIDEA Gray, 1840

Familia Productellidae Schuchert, 1929

Subfamilia Overtoniinae Muir-Wood y Cooper, 1960

Tribu Costispiniferini Muir-Wood y Cooper, 1960

Género Tuberculatella Waterhouse, 1982

Especie tipo: Tuberculatella tubertella Waterhouse, 1982, del Carbonífero tardío de Tailandia.

Distribución geográfica y estratigráfica: Eurasia (Urales, Taimir), Tailandia y Argentina. Carbonífero superiorPérmico inferior.

\section{Diagnosis}

Concha pequeña a mediana, plano-convexa a suavemente cóncavo-convexa, geniculada; espinas escasas dispuestas sobre bases redondeadas en la región posterior y alargadas en la región anterior, dispuestas alternadamente sobre la valva ventral; hoyuelos y espinas muy pequeños y escasos sobre la valva dorsal; interior de la valva ventral con impresiones de los músculos aductores alargadas, impresiones de los músculos diductores estriadas radialmente; interior de la valva dorsal con septo medio largo, aductores anteriores subredondeados sobre una plataforma poco elevada; aristas braquiales suaves.

\section{Discusión}

Recientemente Brunton et al. (1995) y Brunton y Lazarev (1997), en una profunda revisión de la clasificación y evolución del Orden Productida, incluyeron al género Tuberculatella dentro de la Tribu Costispiniferini. Tuberculatella se diferencia exteriormente de Bulahdelia Roberts (Roberts et al., 1976) en la ausencia en ambas valvas de láminas (lamellae) con hileras de espinas anteriormente, en la presencia de espinas huecas en las aurículas y flancos y en las bases de espinas alargadas. Este tipo de bases de espinas también están presentes en los géneros Levipustula Maxwell y Lanipustula Klets (Simanauskas, 1996a). Sin embargo, en Tuberculatella las mismas están presentes sólo en la porción anterior y no en toda la superficie. De Geniculifera Muir-Wood y Cooper, 1960, se diferencia claramente por la presencia de espinas en la valva dorsal.

\section{Tuberculatella peregrina (Reed, 1927)} Fig. 5a-g, Tab. 3

1927 Productus (Marginifera) spinulo-costatus Arbich var. nov. peregrina Reed, en Du Toit, 134, lám. 13, fig. 2. 
1979 “Marginifera” spinulo-costata Arbich var. peregrina Reed; Amos, 77.

1989 Bulahdelia cf. myallensis Roberts; Taboada, 127-128, lám. 2, fig. 9.

1998 Productella sp.; Lech et al., 406-407, figs. 3c a 3e.

Localidad: Quebrada de Eloy, extremo sur de la sierra de Barreal, aproximadamente $3,5 \mathrm{~km}$ al sudeste del pueblo de Barreal, provincia de San Juan, Argentina.

Posición estratigráfica: Parte superior del perfil de la Formación El Paso, intervalo faunístico inferior, niveles 2, 5, 6,7 y 8 .

Material: Tres moldes externos de valvas dorsales (IPI 3313 a y DCG-MLP 309 a y b); un molde externo (IPI 3312) y un molde interno (IPI 3314) de valva ventral; tres moldes internos y externos de valvas ventrales (IPI 3319 a y b, 3316 a y b, DCG-MLP 310 a); dos moldes internos de ambas valvas (DCG-MLP 310 b y c).

\section{Descripción}

Morfología externa: La concha es de tamaño mediano, más ancha que larga. La anchura medida fue de $26 \mathrm{~mm}$ y la longitud de $22 \mathrm{~mm}$ (ver Tab. 3). La línea cardinal es igual o ligeramente menor que la anchura, la cual se mide en la mitad de la longitud de la valva. La valva ventral, convexa y fuertemente incurvada, presenta un seno amplio y suave. Las aurículas están bien delimitadas y portan no menos de tres espinas erectas y huecas. Las aurículas están separadas del disco por una hilera de, por lo menos, tres espinas huecas gruesas, similares a las anteriores. El disco de la valva ventral presenta bases de espinas alargadas anteriormente. Las bases son de $2 \mathrm{~mm}$ de largo y se distribuyen alternadamente. La densidad de las mismas es de 3 en $5 \mathrm{~mm}$ lineales en el centro del disco. La valva dorsal presenta un disco suavemente cóncavo, la geniculación es muy marcada y la cola es larga. El disco de la valva dorsal presenta hoyuelos (pits) correspondientes a las bases de espinas de la valva ventral. Presenta además, escasas espinas muy finas y difíciles de observar. La densidad de las mismas es de aproximadamente 5 en $5 \mathrm{~mm}$ lineales en el centro del disco. El exterior de la concha presenta líneas de crecimiento muy finas y algo sinuosas y

\begin{tabular}{|l|c|c|c|}
\hline IPI & L & $A$ & LC \\
\hline $3316 \mathrm{~b}$ & 13,6 & 18 & - \\
\hline $3313 \mathrm{a}$ & 13,7 & 21,6 & 10 \\
\hline 3314 & - & 20,5 & 9 \\
\hline 3312 & - & 26 & - \\
\hline 3319 & $22 ?$ & 22 & 8 \\
\hline DCG-MLP & & & \\
\hline $309 \mathrm{a}$ & 13,7 & 21,5 & 7 \\
\hline $310 \mathrm{a}$ & - & 22 & 10,5 \\
\hline
\end{tabular}

Tabla 3. Dimensiones en mm de Tuberculatella peregrina (Reed, 1927). A: anchura; L: longitud; LC: longitud de la cola.

Measurements in mm of Tuberculatella peregrina (Reed, 1927). A: width; L: length; LC: length of trail.

también arrugas suaves concéntricas, las cuales están más marcadas en las aurículas. La superficie de la cola, en ambas valvas, es lisa.

Interior de la valva ventral: Las impresiones de los músculos diductores son muy grandes y marcadas. Las mismas son flabeladas y presentan seis costillas angostas de distribución radial, separadas por intervalos del doble del grosor de las costillas. Las impresiones de los músculos aductores son alargadas, subrectangulares y lisas. Estas impresiones están alojadas sobre una plataforma poco elevada, entre las impresiones de los diductores. La superficie interior de la valva ventral, presenta "costillas" muy suaves, radiales, anchas con espacios intercostales muy angostos. La densidad de las mismas es de 5 por cada $\mathrm{mm}$ lineal tomada inmediatamente por delante de las impresiones de los músculos diductores. También se observan aberturas abultadas correspondientes a las espinas huecas. El interior de la cola, al igual que el exterior, es liso.

Interior de la valva dorsal: Presenta un proceso cardinal sesil y bífido, con los lóbulos bien separados. A ambos lados

Figura 5. a-g. Tuberculatella peregrina (Reed, 1927). a, réplica en látex del molde externo de la valva ventral, latex cast of external mould of the ventral valve, IPI 3312, x2,5. b-c, réplica en látex del molde externo e interno de la valva ventral, latex cast of external and internal mould of the ventral valve, IPI 3319a-b, $\mathrm{x} 2$. d, molde externo de valva dorsal, external mould of dorsal valve, DCG-MLP 309b, x2. e, réplica en látex del molde externo de la valva ventral, latex cast of external mould of the ventral valve, IPI 3316b, x2. f, réplica en látex del molde interno de la valva dorsal, latex cast of internal mould of the dorsal valve, DCG-MLP 310b, x2.5. g, réplica en látex del molde interno de la valva dorsal, latex cast of internal mould of the dorsal valve, DCG-MLP 310c, x3. h-i, k-1. Aseptella aff. patriciae Simanauskas. h, molde externo de valva ventral, external mould of ventral valve, IPI 3340a, x4. i, molde externo de valva dorsal, external mould of dorsal valve, IPI 3350, x4. k, molde interno de valva ventral, internal mould of ventral valve, IPI 3339a, x3. I, molde interno de valva dorsal, internal mould of dorsal valve, IPI 3338 , x2. j, m-o. Meristelloidea ind. j, molde interno de valva ventral, internal mould of ventral valve, IPI 2834, $\mathrm{x} 1,5 . \mathbf{m}$, molde interno de valva dorsal, internal mould of dorsal valve, IPI2843, x2. n, vista posterior del molde interno de un ejemplar articulado, internal mould of articulate specimen, posterior view, IPI 2856, x2. o, molde interno de valva ventral, internal mould of ventral valve, IPI $2835, \mathrm{x} 2$. 

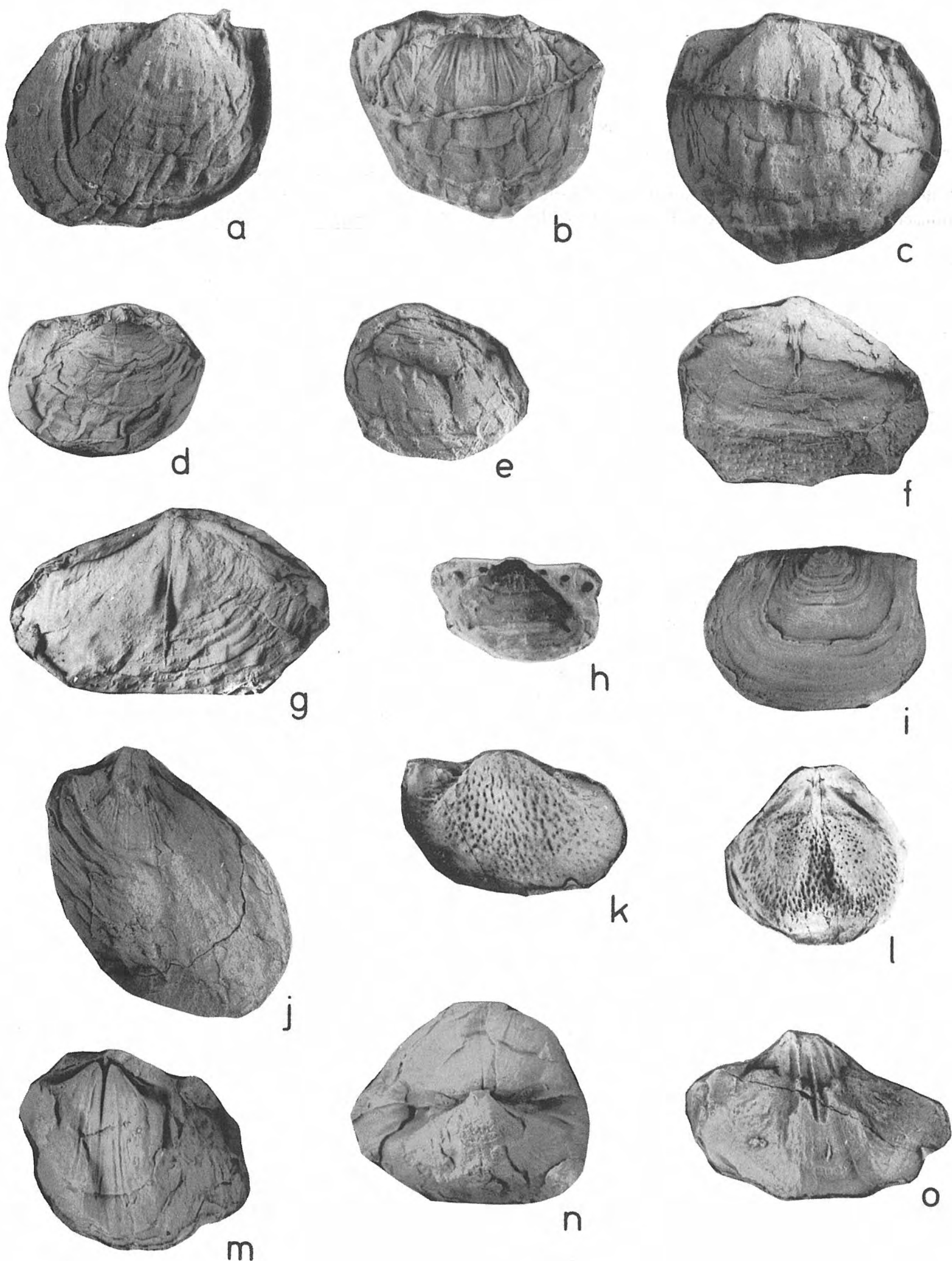
del mismo se observan las aristas cardinales que son subparalelas a la línea cardinal. Por delante del proceso cardinal, se desarrolla una plataforma larga y marcada, la cual se prolonga en un septo medio. La longitud de esta plataforma equivale aproximadamente a la mitad de la longitud del septo. El septo medio es fino, se eleva en su porción media y vuelve a ser bajo en el extremo anterior. El mismo se extiende hasta casi la mitad de la longitud del disco visceral. Las impresiones de los músculos aductores anteriores se encuentran sobre dos plataformas poco elevadas, ubicadas a ambos lados de la mitad posterior del septo medio. Las mismas son en general lisas y en algunos ejemplares suavemente dendríticas. Las impresiones de los músculos aductores posteriores son de forma subtriangular. Las aristas braquiales son finas y están poco marcadas. Las mismas tienen forma de "U" con la abertura apuntando hacia el proceso cardinal.

\section{Comparaciones}

En la descripción e ilustración original de Reed (en Du Toit, 1927) se dan a conocer características morfológicas que permiten asignar los ejemplares aquí estudiados a Productus (Marginifera) spinulo-costatus Arbich var. nov. peregrina. Estas características descriptas por Reed (pág. 134) son la presencia de bases de espinas alargadas, un seno medio ancho y suave y la hilera de espinas entre las aurículas y el disco. La ilustración del único ejemplar estudiado por Reed (Du Toit, 1927, lám. 13, fig. 2) es una reconstrucción parcial del mismo, ya que la valva no está tan completa como en la ilustración, aunque definitivamente se trata del ejemplar $\mathrm{N}^{\circ} 7374$ (Dr. Klinger, South African Museum, comunicación personal). El único ejemplar descrito e ilustrado por Taboada (1989) como Bulahdelia $\mathrm{cf}$. myallensis Roberts, también pertenece a esta especie. El contorno de la concha y la densidad de espinas sobre el vientre y aurículas permiten la reasignación. Con respecto al material descrito e ilustrado por Lech et al. (1998) como Productella sp., también pertenece a Tuberculatella peregrina (Reed, 1927). La ornamentación de la valva ventral, forma de las aurículas, espinas auriculares erectas y tamaño general no difieren del material de El Paso. En comparación con esta especie, Tuberculatella laevicaudata (Amos) del Pérmico inferior de Patagonia (Simanauskas, 1996c) es levemente más alargada y pequeña y casi no presenta seno. Los caracteres internos de la especie patagónica también son menos marcados. Con relación a la ornamentación, la densidad de espinas en ambas valvas es muy similar entre estas dos especies. En otras palabras, la especie patagónica es una forma afín a la precordillerana, aunque más delicada y con ornamentación más suave. Con la especie tipo, Tuberculatella tubertella, comparte el mayor desarrollo del seno, así como la presencia de características internas más marcadas, particularmente las impresiones de los músculos diductores. En este sentido, Tuberculatella peregrina parece ser una forma morfológicamente intermedia entre la especie tipo y Tuberculatella laevicaudata (Amos) del Pérmico de Patagonia.
Familia Productellidae Schuchert, 1929

Subfamilia Plicatiferinae Muir-Wood y Cooper, 1960

Tribu Plicatiferini Muir-Wood y Cooper, 1960

Género Aseptella Martínez Chacón y Winkler Prins, 1977

Especie tipo: Aseptella asturica Martínez Chacón y Winkler Prins, 1977; Formación Ricacabiello, España, Bashkiriense (Martínez Chacón et al., 1985).

\section{Diagnosis}

Concha muy pequeña a pequeña, cóncavo-convexa, con un reborde o cola casi paralela al plano comisural; ornamentada con arrugas suaves sobre la valva ventral y lamelas en la valva dorsal; escasas espinas gruesas dispuestas en dos hileras a ambos lados, una paralela a la línea cardinal y la otra cruzando el disco desde el umbón hasta la región anterolateral de la valva ventral; valva dorsal sin espinas, con escasos hoyuelos.

Distribución geográfica y estratigráfica: Europa (NO de España) y Sudamérica (Argentina). Carbonífero inferior Pérmico inferior.

\section{Aseptella aff. patriciae Simanauskas, 1996}

Fig. 5h-i, k-1

1998 Aseptella ? sp., Lech et al., 405- 406, fig. $3 \mathrm{~h}$.

Localidad: Quebrada de Eloy, extremo sur de la sierra de Barreal, aproximadamente $3,5 \mathrm{~km}$ al sudeste del pueblo de Barreal, provincia de San Juan, Argentina.

Posición estratigráfica: Parte superior del perfil de la Formación El Paso, intervalo faunístico inferior, nivel 8.

\section{Observaciones}

Los ejemplares de Aseptella aff. patriciae refigurados en este trabajo ya fueron descriptos y figurados previamente, dentro del marco de un estudio del género Aseptella en Argentina (Cisterna y Simanauskas, 1999, fig. 2a-1). En relación con Aseptella? sp. descripta por Lech et al. (1998, pág. 405, fig. 3h), teniendo en cuenta la ornamentación de la valva y sus dimensiones, la misma pertenece sin dudas a la especie presente en la Formación El Paso.

ORDEN ATHYRIDIDA Boucot, Johnson y Stanton, 1964 SUBORDEN ATHYRIDIDINA Boucot, Johnson y Stanton, 1964

Superfamilia MERISTELLOIDEA Waagen, 1883

\section{Meristelloidea ind.}

Fig. 5j, m-o

Localidad: Quebrada de Eloy, extremo sur de la sierra de Barreal, aproximadamente $3,5 \mathrm{~km}$ al sudeste del pueblo de Barreal, provincia de San Juan, Argentina.

Posición estratigráfica: Parte superior del perfil de la Formación El Paso, intervalo faunístico inferior, nivel 8. 
Material: ocho moldes internos de valvas ventrales, IPI 2834-2841; diez moldes internos de valvas dorsales, IPI 2843-2851; dos moldes internos correspondientes a ejemplares articulados, IPI 2855-2856.

\section{Descripción}

Especímenes grandes, lisos, de contorno subtriangular alargado, con umbón ventral moderadamente prominente. La superficie exhibe conspicuas líneas de crecimiento concéntricas. El interior de la valva ventral exhibe láminas dentales cortas y ligeramente divergentes. El campo muscular está fuertemente impreso y se ensancha anteriormente, en donde las huellas de los músculos diductores adquieren una disposición en forma de abanico. En la parte posterior del campo muscular se observa una elevación central alargada con una depresión media. Las impresiones ováricas rodean al campo muscular en su parte anterior. El interior de la valva dorsal presenta un fuerte septo medio que se extiende hasta aproximadamente la mitad de la longitud de la valva y un septalio conspicuo sustentado por el septo medio. Otros caracteres internos no se han observado.

\section{Discusión}

El material descripto, integrado prácticamente en su totalidad por moldes bien conservados, ha sido asignado de forma preliminar a Meristelloidea ind.

La diferencia fundamental entre las dos grandes superfamilias Meristelloidea y Athyridoidea que integran el Orden Athyridida, está basada en la presencia o no de septalio en la valva dorsal (Alvarez et al., 1998). Debido a la presencia de esa estructura, el material aquí analizado podría integrarse en la Superfamilia Meristelloidea. Esta superfamilia está ampliamente representada en el Paleozoico inferior y sólo llega al Carbonífero con la Familia Meristidae Hall y Clarke. Sin embargo, aunque en términos generales el material aquí analizado podría incluirse en esta familia, no se ha encontrado concordancia con ninguno de los géneros en ella integrados. De manera tal que nuestro material podría corresponder a un nuevo género dentro la familia Meristidae, que precisamente en Argentina alcanzaría los niveles más jóvenes del Carbonífero o, incluso, el Pérmico.

\section{AGRADECIMIENTOS}

Los autores agradecen al Dr. Mauricio Martínez y a la geóloga Andrea Sterren (Universidad Nacional de Córdoba), quienes colaboraron en las tareas de campo y en la recolección del material fósil aquí estudiado. Asimismo, al Dr. Fernando Álvarez (Universidad de Oviedo) por sus opiniones vertidas en relación con los braquiópodos atíridos, como así también, al Sr. Derek Ohland (Collections Manager, Division of Earth Sciences, South African Museum) y al Dr. Herbert C. Klinger, de la misma institución, por la información y opiniones acerca del material tipo correspondiente a Tuberculatella peregrina. Desean también expresar su agradecimiento al Dr. Luis Benedetto (Universidad Nacional de Córdoba) por el apoyo logístico en las tareas de campo. El presente trabajo fue subsidiado parcialmente por el CONICET, dentro del marco del Proyecto de Investigación "Braquiópodos del Paleozoico superior de la cuenca Calingasta-Uspallata, provincias de San Juan y Mendoza". Finalmente, los autores hacen extensivo su agradecimiento a los árbitros de este trabajo, Drs. Jenaro García Alcalde (Universidad de Oviedo) y Cor Winkler Prins (Nationaal Natuurhistorisch Museum, Leiden), como así también a las correcciones editoriales por parte de la Dra. María Luisa Martínez Chacón, las cuales mejoran la calidad del mismo.

\section{BIBLIOGRAFÍA}

Alvarez, F., Jia-Yu, R. and Boucot, A.J. 1998. The Classification of Athyridid Brachiopods. Journal of Paleontology, 72, 827-855.

Amos, A.J. 1979. Guía Paleontológica Argentina. Parte I: Paleozoico, faunas carboníferas y pérmicas. Publicación del CONICET, 1-154.

Amos, A.J. y Rolleri, E.O. 1965. El Carbonífero medio en el Valle Calingasta- Uspallata (San Juan-Mendoza). Boletín de Informes Petroleros, 368.

Archbold, N.W. 1981. Permian Brachiopods from the Western Irian Jaya, Indonesia. Geological Research and Development Center, Paleontology Series, 2, 1-25.

Azcuy, C.L., Sabattini N. and Taboada, A. 1990. Advances in Lower Carboniferous zonation of Argentina. Courier Forshchung-Institut Senckenberg, 130, 207-210.

Banchig, A.L., Milana, J.P. y Lech, R.R. 1997. Formación Casa de Piedra, una nueva unidad paleozoica glacimarina en la Sierra del Tontal, Precordillera Occidental, San Juan. Actas de las Segundas Jornadas de Geología de Precordillera, 1, 16-21.

Brunton, C.H.C. and Lazarev, S.S. 1997. Evolution and Classification of the Productellidae (Productida), Upper Paleozoic Brachiopods. Journal of Paleontology, 71, 381-394.

Brunton, C.H.C., Lazarev, S.S. and Grant, R.E. 1995. A review and new classification of the brachiopod Order Productida. Palaeontology, 38, 915-936.

Cisterna, G.A. 1997. Taxonomía y Paleoecología de los Rhynchonellida, Spiriferida y Strophomenida (Brachiopoda) del Paleozoico tardio de las cuencas Río Blanco y Calingasta-Uspallata, provincias de La Rioja y San Juan. Tesis Doctoral. Facultad de Ciencias Exactas, Físicas y Naturales de la Universidad Nacional de Córdoba. Inédito, 1-173.

Cisterna, G.A. y Simanauskas, T. 1999. Aseptella (Brachiopoda) en el Paleozoico tardío de Argentina. Revista Española de Paleontología, 14, 117-122.

Cooper, G.A. and Grant, R.E. 1969. New Permian Brachiopods from West Texas, Smithsonian Contributions to Paleobiology, 1, 20 pp.

Cooper, G.A. and Grant, R.E. 1975. Permian Brachiopods of West Texas III. Smithsonian Contributions to Paleobiology, 19, 795-1921.

Cvancara, A. M., 1958. Invertebrate fossils from the Lower Carboniferous of New South Wales. Journal of Paleontology, 32, 846-888. 
Du Toit, A. L. 1927. A geological comparison of south America with south Africa. With palaeontological contribution by F.R. Cowper Reed. Carnegie Institution Publications, 381, 1-157.

González, C.R. 1990. Development of the Late Paleozoic glaciations of the South American Gondwana in Western Argentina. Palaeogeography, Palaeoclimatology, Palaeoecology, 79, 275-287.

González, C.R. 1993. Late Paleozoic Faunal Succession in Argentina. Comptes Rendus XII ICC-P, Buenos Aires, 1991, 1, 537-550.

González, C.R. 1999. Permo-Carboniferous faunas and provincialism in the South American Gondwanan region. Newsletter on Carboniferous Stratigraphy, 17, 12-13.

González, C.R., Taboada, A.C., Díaz Saravia, P. y Aredes, M. 1995. El Carbónico del sector noroccidental de la Provincia del Chubut. Revista de la Asociación Geológica Argentina, 50, 40-46.

Grant, R.E. 1976. Permian Brachiopods from Southern Thailand. Journal of Paleontology, Memoir 9, 50 (2), 1269.

Lech, R.R., Milana, J.P. y Banchig, A. 1998. Braquiópodos carboníferos en la Sierra del Tontal, provincia de San Juan, Argentina. Ameghiniana, 35, 405-413.

Léveillé, C. 1835. Aperçu Géologique de quelques Localités très riches en Coquilles sur les Frontières de France et de Belgique. Société Géologique de France, 2, 29-40.

Martínez, M., Cisterna, G.A. and Simanauskas, T. 1998. Sedimentary evolution and biostratigraphic framework of Carboniferous glaciation in Barreal Hill, CalingastaUspallata basin. VII Congreso Argentino de Paleontología y Bioestratigrafía, (Bahía Blanca), pág. 91.

Martínez Chacón, M.L. and Winkler Prins, C.F. 1977. A Namurian brachiopod fauna from Meré (Province of Oviedo, Spain). Scripta Geologica, 39, 1-67.

Martínez Chacón, M.L., Menéndez-Alvarez, J.R., Sánchez de Posada, L.C. y Truyols, J. 1985. Aportaciones al conocimiento de la Formación Ricacabiello (Carbonífero de la Zona Cantábrica, $\mathrm{N}$ de España) y su contenido paleontológico. Trabajos de Geología. Universidad de Oviedo, 15, 53-65.

Maxwell, W.G. 1961. Lower Carboniferous brachiopod faunas from Old Cannindah, Queensland. Journal of Paleontology, 35, 82-103.

Mésigos, M. 1953. El Paleozoico Superior de Barreal y su continuación austral, Sierra de Barreal (Provincia de San Juan). Revista de la Asociación Geológica Argentina, 8 (2), 65-109.

Muir-Wood, H. and Cooper, G.A. 1960. Morphology, Classification and Life Habits of the Productoidea (Brachiopoda). The Geological Society of América,
Memoir, 81, 447 pp.

Newell, N.D., Chronic, J. and Roberts, J. 1953. Upper Paleozoic of Perú. Memoir of the Geology Society of América, 58, 1-276.

Oehlert, D.P. 1890. Notes sur différentes groupes établis dans le genre Orthis et en particulier sur Rhipidomella Oehlert (= Rhipidomys Oehlert). Journal Conchyliologie, 3 (30), 366-374.

Racheboeuf, P.R. 1998. The Chonetoidean Brachiopods. A revised and updated systematic and bibliographic catalogue. Documents des Laboratoires de Géologie Lyon, 148, 178 pp.

Roberts, J., Hunt, J.W. and Thompson, D.M. 1976. Late Carboniferous marine invertebrate zones of eastern Australia. Alcheringa, 1, 197-225.

Roberts, J., Claoue-Long, J., Jones, P.J. and Foster, C.B. 1995. SHRIMP zircon age control of Gondwanan sequences in Late Carboniferous and Early Permian Australia. In: Non-biostratigraphical Methods of Dating and Correlation (Eds. R.E. Dunay and E.A. Hailwood). Geological Society Special Publication, 89, 145-174.

Simanauskas, T. 1996a. Una nueva especie de Lanipustula (Productoidea, Brachiopoda) del Paleozoico Superior de Argentina. Ameghiniana, 33, 301-305.

Simanauskas, T. 1996b. Un supuesto Chonetoidea del Pérmico de Patagonia, reasignado a un género de Productoidea (Brachiopoda). Ameghiniana, 33, 349-351.

Simanauskas, T. 1996c. Braquiópodos (Productoidea) del Pérmico de la Cuenca Tepuel-Genoa, Chubut, Argentina. Ameghiniana, 33, 377-383.

Simanauskas, T. y Sabattini, N. 1997. Bioestratigrafía del Paleozoico Superior marino de la Cuenca Tepuel-Genoa, Chubut, Argentina. Ameghiniana, 34, 49-60.

Taboada, A.C. 1989. La fauna de la Formación El Paso, Carbonífero inferior de la Precordillera sanjuanina. Acta Geológica Lilloana, 17, 113-129.

Taboada, A.C. 1997. Bioestratigrafía del Carbonífero marino del valle de Calingasta-Uspallata, provincias de San Juan y Mendoza. Ameghiniana, 34, 215-246.

Taboada, A.C. 1998. Dos nuevas especies de Linoproductidae (Brachiopoda) y algunas consideraciones sobre el Neopaleozoico sedimentario de las cercanías de Uspallata, Argentina. Acta Geológica Lilloana, 18, 69-80.

Waterhouse, J.B. 1982. New Carboniferous brachiopod genera from Huai Bun Nak, North-east Thailand. Paläontologische Zeitschrift, 56 (1-2), 39-52.

Winkler Prins, C.F. and Martínez Chacón, M.L. 1998. A new species of the productid brachiopod Aseptella from the Lower Carboniferous of the Cantabrian Mountains (Spain). Revista Española de Paleontología, 13, 243249. 